\title{
Analgesic Efficacy of Ultrasound Guided Quadratus Lumborum Block and FasciaI liaca Compartment Block for Patient Schedualed for Dynamic Hip Screw Surgery
} M.G.Elsayed, M.B.Hasanin, R.Kh.Kamel and M.H.Abdelrahman

Anaesthesia and Surgical Intensive Care, Dept., Faculty of Medicine, Benha Univ., Benha, Egypt E-Mail:maha.gamil@fmed.bu.edu.eg

\begin{abstract}
After complete hyparthroplasty, postoperative discomfort is a significant issue. In these patients, many methods are often utilised in postoperative analgesia, including neuraxial analgesia, intravenous analgesia and peripheral neuron blocks. The benefit of peripheral nerveblocks is phytosanitary treatment, reduced engine impairment and fewer systemic effects. Common peripheral blocks of nerve for hip operations include suprainguinal fasciailiacablock(FIB) and lumbar plexusblock. The Quadratus lumborum block (QL) is a newly designed lower abdominal and hip chirurgical block with high performance. Seventy patients between 8-70 years of age with ASA physical status I-III were enrolled in the research and were scheduled for dynamic hip screw surgery under subarachnoid block (SAB) hip. Patients were randomly assigned in one of two groups using the hidden closed envelope method: group of FICBs $(n=35)$ : this group receives a suprainguinal iliac fascia block, the end of the injection is deep to the fascia iliaca and the lateral portion of the iliacus muscle above the iliacus. $30 \mathrm{~mL}$ of 0.25 percent of Bupivacaine is injected gradually beneath the fascial plane and each $5 \mathrm{ml}$ is absorbed after negative aspiration. QLB Group ( $n=35)$ : the group got ultrasonically-guided lumborum block transmuscular quadratus; (QLB anterior or QLB III) a needle tip between the main muscle of the psoas and the lumborum muscles of the quadratus is inserted. After negative aspiration, $30 \mathrm{~mL}$ of $0.25 \%$ bupivacaine is gradually administered into the fascial plane, each $5 \mathrm{ml}$ aspiration. Both FIB and transmuscular QLB performed in the first 24 hour postopérative and comparable analgesic duration following hip arthroplasty operations with similar static and dynamic VAS. Both single shot blocks FIB and QLBT offer excellent postoperative analgesia afterTHA. FIB demonstrated a little reduced intake of morphine over 24 hours.
\end{abstract}

Keywords: Hiparthroplasty, Fasciailiacablock, Quadratus lumborum block, Effectivepostoperative analgesia.

\section{Introduction}

The regional anaesthetic technique Quadratuslumborum (QL) block was first published in 2007 as a method for abdominal surgical analgesia. Several years later it was discovered that this block also may provide analgesia to the hip and several studies showed that femoral neck fracture and hip arthroplasty were effective The anatomical target of the QL block is the anterolateral dimension of the QL muscle in the area between the QL fascia and the posterior endoabdominal fascia as first defined by Blanco and McDonnell [1, 2] Local anaesthetic injected into this region is thought to travel medially as far as T6 and L3, to inhibit root roots as they leave the intervertebral foramine. An alternate theory is that there is a subsequent blocking of the vertebrae in this posterior fascial injection plane, blocking the abdominal and lumbar plexal neurons after the muscle has left Psoas [3]. Peripheral nerve blockage has been proven to enhance pain rates and decrease morphine use in patients having hip operation. The compartment block (FICB) of Fascia iliaca may cause sensory blockage of the major nerves that generate hip pain [4]. Recently, a proposal was made for a fascia iliaca compartmewntal block to prevent problems by remotely anaesthetizing the femoral nerve from the main neurovascular structures and achieving sufficient analgesia[5]. Dynamic hip screw (DHS) surgery for fracture femure is frequent surgical treatment methods for degenerative and traumatic illnesses. A majority of patients, following dynamic hip screw operation, typically suffer moderate to severe postoperative pain [6]. Postoperative pain management has a significant effect on earlier ambulation, physiotherapy start, and improved recovery. In addition, good pain management reduces the duration of hospitalisation and the risk of thrombotic events that increase patient satisfaction. Multiple analgesic methods, including intravenous opioid, epidural analgesia and peripheral nerve block, have been suggested. Each has its own limits [7]. Patients suffering from fracture femur may be exposed to opioids for extended periods of time and may be at high risk of associated opioid hazard, especially chronic users of opioids who take opioids pre-operatively and chronically [8]. Systemic use of opioids is linked with patient discontent, such as nausea, vibration, constipation, urine retention, increased clinical outcomes in orthopaedic surgery and higher early postoperative opioid usage is associated with longer duration. [9]

\section{Materials and Methods Patients}

The research was conducted at the Benha Surgical Hospital University, Egypt. The research was authorised by Benha University Hospitals Committee on Institutional Ethics. Each patient received written informed consent at the preoperative appointment. We have registered patients over 8 years old with a physical status classification score from 1 to IV from the American Society of Anesthesiologists for elective Elective dynamic hip screw surgery, body mass index less than $40 \mathrm{~kg} / \mathrm{m} 2$. 
The exclusion criteria were patient refusal, who cannot agree to a site infection of blocks less than 8 years old, coagulopathy and body mass index larger than $40 \mathrm{~kg} / \mathrm{m} 2$. In preanesthetic assessment of patients each patient was given demographic data and instruction was given regarding VAS painscore $(0-00$ $\mathrm{mm}$ ) in the visual analogue scale (with $0=$ no pain and $00=$ worst understandable pain) and the nervous block procedural specifics.

\subsection{Anesthesia}

Patients were taken to the operating room and were monitored by ASA standard monitors : ECG, NIAB , pulse oximetry:

\subsection{Technique of suprainguinal fascia iliaca block (FIB)}

At the supine position, the high-frequency linear General Electric transducer, GE, LOGIQ P5 ultrasonic 6-3 MHz machine and the anterior superior iliac column were placed in the sagittal planetoidentifier and the transducer were moved to the medium of the fascia iliaca, iliopsoas, sartorias and interior oblique muscles. A 00mm, 2-G block needle tip was placed further into the fascia iliaca and above the iliacus muscle using an in-plan approach. $30 \mathrm{~mL}$ of $0.25 \%$ bupivacaine were administered progressively beneath the fascial plane after a negative injection, each aspirating $5 \mathrm{~mL} \quad 2.3$ Transmuscular quadratus lumborum block technique (QLB3, previous QLB):

The patient was on the side. A convex lowfrequency probe was vertically connected above the iliac crest and an anterior direction was introduced into the plane from the back of the convex probe through the quadratus lumborum with a $2 \mathrm{G}$ nonstimulating block needle. The point of the needle was inserted between the main muscle psoas and the lumbor muscle quadratus. Following negative aspiration, $30 \mathrm{~mL} 0.25 \%$ bupivacain was injected into the fascial plane gradually and each $5 \mathrm{ml}$ was sucked in.

\subsection{Subarachnoid block (SAB):}

Patients received $\mathrm{SAB}$ after 5 minutes of block, using $25 \mathrm{~g}$ spinal needle in the lateral position. Fifteen milligrams of hyperbaric bupivacaine was administered at L3-L4 or at L4-L5 interspace in addition to $25 \mathrm{mcg}$ fentanyl with rapid crystalloid coload. Spinal anesthesia was considered successful when a bilateral block to T2 was achieved; the block was assessed 0 -minutes after the intrathecal injection by loss of cold (cold ice) and pin prick (a 23-gauge needle) sensations. If spinal anesthesia failed, general anesthesia was administered and the patient was excluded from this study.

\subsection{Postoperative care}

Patients were transferred top ostanestheticcare unitfor2hoursafteranesthesia emergence. The patients will be discharged from the PACU after fulfilling the discharge criteria based on the modified Aldrete score > 9.Patients received analgesics according to local institutional protocol as the following: (paracetamol gmIVinfusion/8hrs, ketorolac $30 \mathrm{mg} \mathrm{IM} / 2 \mathrm{hrs}$ ) as 2 components of multi modalanesthesiaregimen for postoperative pain control.\Apostoperativerescueanalgesia with intravenous morphineper atitration protocol $(3 \mathrm{mg}$ morphine sulfate IV as a bolus dose that could be repeated every 5 minutes with a maximum dose of $5 \mathrm{mg}$ per 4 hours or $45 \mathrm{mg}$ per 24 hours) was employed if visual analog pain scale $(\mathrm{VAS})>4$.

\subsection{Outcome Measures}

Theprimary outcome measure at the commencement of the study was total postoperative morphine consumption in the first 24 hours.Secondary outcome measures VAS scores at five different time-points (0 "on arrival", 6th hours, 2th hours, 8th hours and 24th hours)

\subsection{Statistical ianalysis}

$\mathrm{G} *$ Power $\odot$

softwareversion3.7.

Thesamplesizecalculated (Instituteof experimental psychology,HeinrichHeineUniversity,Dusseldorf , Germany). Each group should include a group of 35 individuals at drop out rates of 0 percent, depending on prior study findings with an error of 0.05 on two sides (two tails), type I and an impact of 80 percent, effect size (d) factor (0.8). The results of the two groups will be compared using the Social Science Statistical Package (SPSS Inc., Chicago, IL, USA). Use of Student's t-Tests to present the normally distributed parametric numerical data as a mean $( \pm$ SD) and compare differences between groups, the non-parametric data as a medium or interquartile area is presented and differences between groups will be compared using Mann-Whitney U-test, the category data is presented as number and percentage and the comparison between groups will be performed. The findings are statistically significant if the $\mathrm{p}$ value is less than 0.05 and the confidence interval is $95 \%$.

\section{Results}

This study was conducted on 70 patients scheduled for dynamic hip screw surgery at Benha University Hospital. Patients were divided into two groups: Group A: Thirty-five patients received FICB plus conventional opioid analgesics.Group B: Thirty-five patients received QL plus conventional opioid analgesics.There were no significant differences between both groups as regard age ( $\mathrm{P}$ value $=0.359)$, weight $(\mathrm{P}$-value $=0.36), \mathrm{BMI}(\mathrm{P}-$ value $=0.745)$, and height $(\mathrm{P}-$ value $=0.529)$ Tabel . 
Table (1) General characteristic inboth group.

\begin{tabular}{llccc}
\hline & Group A & $\begin{array}{c}\text { Group B } \\
(\mathbf{n}=35)\end{array}$ & P-value \\
\hline Age (years) & Mean \pm SSD & $52 \pm 8$ & $50 \pm 7$ & 0.359 \\
Weight (kg) & Mean \pm SD & $8 \pm 6$ & $83 \pm 6$ & 0.36 \\
BMI & Mean \pm SD & $25.7 \pm .6$ & $25.9 \pm 3$ & 0.745 \\
Height (cm) & Mean \pm SD & $78 \pm 6$ & $79 \pm 5$ & 0.529 \\
\hline
\end{tabular}

Tabel (2) Duration of surgery and morphine consumption.

\begin{tabular}{llccc}
\hline & & $\begin{array}{c}\text { Group A } \\
(\mathbf{n = 3 5})\end{array}$ & $\begin{array}{c}\text { Group B } \\
(\mathbf{n = 3 5})\end{array}$ & P-value \\
\hline Duration of surgery $(\mathbf{m i n})$ & Mean \pm SD & $34 \pm 7$ & $30 \pm 6$ & 0.320 \\
Morphine Consumption & Mean \pm SD & $9 \pm 6$ & $25 \pm 6$ & $<0.00$ \\
\hline
\end{tabular}

Tabel (3) VASR in both groups.

\begin{tabular}{llccc}
\hline & & $\begin{array}{c}\text { Group A } \\
(\mathbf{n = 3 5})\end{array}$ & $\begin{array}{c}\text { Group B } \\
(\mathbf{n = 3 5})\end{array}$ & P-value \\
\hline At 0 time & Median (range) & $2(0-3)$ & $2(0-4)$ & 0.007 \\
At 6 hours & Median (range) & $3(-4)$ & $4(2-5)$ & 0.00 \\
At 2 hours & Median (range) & $2(-5)$ & $3(-5)$ & $<0.00$ \\
At 8 hours & Median (range) & $2(-4)$ & $3(2-5)$ & $<0.00$ \\
At24 hours & Median (range) & $2(-4)$ & $4(2-5)$ & $<0.00$ \\
\hline
\end{tabular}

Duration of surgery and morphine consumption (Tabel 2)

There was no significant difference between both groups as regards duration of surgery $(\mathrm{P}$-value = 0.32).Morphine

consumption was significantly higher in group B (25) compared to group A (9); P-value was $<0.001$.

\section{VASR in both groups (Tabel 3)}

At 0 time, VASR showed a significant difference between groups with a higher range in group B $(0-$ 4) than group $\mathrm{A}(0-3)$; P-value was 0.007 . At 6 hours, median VASR was significantly higher in group B (4) than group A (3); P-value was 0.001 . At 2 hours, median VASR was significantly higher in group B (3) than group A (2); P-value was $<0.001$. At 8 hours, median VASR was significantly higher in group B (3) than group A (2); P-value was $<0.001$. At 24 hours, median VASR was significantly higher in group B (4) than group A (2); P-value was $<0.001$

\section{Discussion}

The patient experienced moderate or even severe pain after total hip arthroplasty (THA), which severely affects the early postoperative recovery. The objective of this research was to examine the analgesic effectiveness of ultrasound-guided lumborum quadratus (T-QLB) vs fascia iliaca compared to senior THA patients.

Perioperative and early postoperative pain remains one of the most frequently mentioned challenges for hip arthroplasty, adequate treatment after the procedure is linked with increased patient satisfaction, earlier mobilisation, decreased pain- related medication requirements and overall better results [1].

Regional anaesthetic has been widely utilised in hip surgery and in arthroplasty surgical operations. In order to identify the most effective analgesic technique, research evaluating the efficacy of the QL block for various methods have started. A recent comparative research has shown a substantial reduction in the duration of hospital stay after total hip arthroplasty compared to no block in individuals receiving QL blocks.

The innervation of the hip is complicated and has contributions from numerous nervous components. Birnbaum et al. found that the nerves implicated in THA incision pain primarily included a subcostal nerve, an iliohypogastric nerve, an ilioinguinal nervous, a femoral nerve and a sciatical nerve block [2]. In this research, both FIB and transmuscular QLB were shown to be efficient in delivering early postoperative analgesia after hip arthroplasty. In the first 24 hours postoperatively, FIB exhibited reduced opioid use. The newest research has also shown that the femoral nerve, which dominates the hip joint, is positioned in a higher position. And the placement of the inguinal ligament on the lateral femoral skin nerve has anatomical diversity [3]. It is thus difficult to fulfil the needs of patients to conduct single-shoot peripheral nerve blocks such as lumbar plexus, sacred plexus, Fascia iliaca block and quadratus lumborum block. The lumbar plexus block has, on the other hand, a significant risk for haemorrhage and nerve damage, since the tip of the needle should be advanced closely to the nerves [4]. 
The fascial plane block method is more protected against conventional peripheral nerve blocks (like the lumbar plexus block) and causes analgesic blocks primarily via local anaesthesia along the fascia plane spreading to its associated nerves (nerve roots) [5]. A previous and safer method to the lumbar plexus block may be regarded as the FIB. It results in femoral, shuttering, and lateral femoral neuron blocks (3-in- block). The suprainguinal technique for FIB increases the probability of blocking the three nerves with a single local anaesthetic injection [2]. QLB has 4 different blocks in respect to the muscle of the needle tip: lateral, posterior, anterior or intramuscular. The tip of the needle lies between the QL and psoas in the flat for anterior or trans-muscular QLB and may be done at various levels, such as L2, L4 and Subcostal [6].

A relatively recent block is transmuscular QLB. The exact mechanism via which the QLB produces hip joint analgesia is not completely known. Various processes may explain QLB's analgesic impact on our patients. (2) the lateral propagation of the local anaesthetic into the spaces of the thoracic and lumbar paravertebral; (3) the potentially spreading of the pleasant lumbar to the lumbar plexus via the fascial layer between the two-thirds of the anterior and the third of the posterior one third of the muscle of a psoa [7]. The actual analgesic mechanism offered by the previous QLB has not yet been completely elucidated. Most of the transmuscular QLB cadaveric experiments revealed that direct teal spreads to the roots and branches of the lumbar plexus [8]. Despite these rare instances, several publications define $\mathrm{QL}$ blocks as an engine-saving technique of localised analgesics and no lower extremity weaknesses were observed in patients in our research. The most effective methods of analgesia must be identified and what might be catastrophic consequences minimized [9]. Cutaneous sensation of the majority of hip surgical wounds from the lateral thigh neuron (L-3), which is inhibited efficiently in suprainguinal FICB. In the case of sensory blockage of QLB, T2L2dermatomas are often included; L3 is rarely included; L4 is normally [20]. This may account for the lower postoperative morphine need in the FIB group than in the QLB group. The timing of the initial analgesic request was similar in both groups, although FIB patients had less morphine than QLB patients. In the QLB group, Quadriceps motor power is better conserved than in the FIB group. The number of patients who had pain during spinal anaesthesia and the hemodynamic characteristics in both blocks after 5 minutes, which were lower in the QLB Group than in FIB owing to a relatively earlier action. With regards to postoperational motor function, most clinical studies on transmuscular QLB did not show hip and knee motor weakness and this may be caused not only by diluted local anaesthesia but also by the typical sparing of the underlying lumbar roots [20]. On the other hand, the weakening of quadriceps is described as a FIB consequence because the femoral nerve is consistently blocked. In case of continuous FIB it is advisable to cease infusions hours before expected mobility so that they do not impede postoperatively [2]. This research simplified the placement of the QLB transmuscular patients under spinal anaesthesia; supplied the supraninguinal FIB with similar analgesic length; and enhanced the maintenance of the motor quadriceps than the suprainguinal FIB. The overall postoperative morphine need in transmuscular QLB was somewhat greater than in suprainguinal FIB. The research supports the analgesic effectiveness of some selective regional methods, for example, lumbar plexus block, FIB, and pericapsular injection, although there is presently no agreement on any single approach or combination of techniques superior than THA [22]. We were able to compare quadratus lumborum versus fascia iliaca blockiin 36 hip arthroplastic patients, and found $24 \mathrm{hrs}$ mean morphine use with fascia iliaca group to be $6 \mathrm{mg}$ lower than quadratus lumborum block group (8 mg) [23], according to Nassar et al. Another research was compatible with our Hashmi et al investigation, which showed TQL did not provide higher or less motor blocks than FICB in patients who are subjected to optional hip replacement surgery [24]. However, other research had contradictory findings. Kukreja et al. found that QLB offers efficient analgesics after hip arthroplasty, [20] whereafter transmuscular QLB, Aoyama et al. failed to see a consistent sensory blocking in its lumbar nerves. Our design of our research varied from that of these two randomised controlled studies [25]. Kukreja et al. contrasted QLB by means of a control (no block) regime, Aoyama et al. compared a continuous QLB to a femoral nerve block. In addition, epinephrine has been utilised for both blocks as an adjuvant to the local anaesthetic combination. In a research conducted by Blackwell et al. to evaluate the effectiveness of Quadratus Lumborum block against Femoral Nerve and Fascia Iliaca blocks in hip arthroscopy, they discovered that the intake of opioids in the quadratus lumborum groups is lower than the intraocular fascia iliaca group. Fascia iliaca blocks, compared to QL block with femoral nerve block and FICB, for hip arthroscopy; and Parras and Blanco, showed that patients with the QL block had reduced perioperative opioid needs and a gradient of analogous visual values in the first 24 hours following Hip arthroscopy [27]. Complications of femoral nerve and fascia iliac are widely established. Quadriceps inhibition resulting to falling and persistent sensory defects include femoral nerve problems and fascia-liac block issues. In fact, postoperative falls in femoral nerve blocks may reach 22 per cent [28]. Potter et al. observed that cutaneous numbness remained in 26 per cent of the patients throughout the first clinical follow-up. Despite the generally favourable results, QL blocks are not risk-free [28]. Ueshima and 
Hiroshi discovered that up to 9 percent of patients experienced a certain quadricep weakness after an aftercutting QL block in all QL methods, even if the mechanism was not apparent and the muscle weakness duration was not indicated. In a QL reporting block for gynaecological laparoscopy, a patient had similar weakness, resulting in unexpected hospitalisation [29]. Postoperative morphine use in the FIB group was slightly lower than in QLB in the current research. The blocking of L2-3 dermatomas, particularly the lateral femoral cutaneous nerve in the FIB, which is a crucial cutaneous sensation for hip surgical incisions may be theoretically explained while the L3 is not blocked consistently in the QLB

\section{Conclusion}

Both single shot blocks, namely suprainguinal FIB and transmuscular QLB, provided effective postoperative analgesia after THA. FIB showed slightly lower 24-hour morphineconsumption

\section{References}

[1] L.La Colla, A.Uskova, B.Ben-David. Singleshot quadratus lumbo- rum block for postoperative analgesia after minimally invasive hip arthroplasty: a new alternative to continuous lumbar plexus block? Reg Anesth Pain Med.vol.42,pp. 25-6,2017.

[2] M. Von Korff, MV.Korff, K. Saunders .De facto long-term opioid therapy for noncancer pain. Clin J Pain.vol.24,pp. 52-7, 2008.

[3] MM.Hockett, S.Hembrador,

Lee.Continuous quadratus lum- borum block for postoperative pain in total hip arthroplasty: a case report. Case Rep.vol.7,pp.29-3, 2016

[4] M.Stevens, G.Harrison, M.McGrail: A modified fascia iliaca compartment block has significant morphine-sparing effects. Anaesth Int Care.vol. 35,pp.949-52,2007.

[5] LQ.Deng, LN. Hou, FX. Song. Effect of preemptive analgesia by continuous femoral nerve block on early postoperative cognitive function following total knee arthroplasty in elderly patients. Exp Ther Med.vol.3,pp.592-7,2017.

[6] JF. Li, H. Li, H. Zhao. Combined use of intravenous and topical versus intravenous tranexamic acid in primary total knee and hip arthroplasty: a meta-analysis of randomised controlled trials. J Orthop Surg Res 2017;2:22.

[7] TF. Bendtsen, B. Moriggl, V. Chan. The optimal analgesic block for total knee arthroplasty. Reg Anesth Pain Med.vol.4,pp.79,2016.

[8] CD. Soelberg, RE. Brown, D. Du Vivier. The US opioid crisis: current federal and state legal issues. Anesth Analg.vol.25,pp. 675-8,2017.

[9] BJ. Morris, HR. Mir. The opioid epidemic: impact on orthopaedic surgery. J Am Acad Orthop Surg.vol.23,pp. 267-7,2015.
[10]JJ. Shin, CL. Mccrum, CS. Mauro, D. Vyas.Pain management after hip arthroscopy: Systematic review of randomized controlled trials and cohort studies. Am J Sports Med.vol.46,pp.3288-98, 2017.

[11] MS. Green , CR. Hoffman, U. Iqbal, OO. Ives, B. Hurd .Transmuscular quadratus lumborum block reduces length of stay in patients receiving total hip arthroplasty. Anesth Pain Med.vol.8,pp.80-233, 2018.

[12] K. Birnbaum, A. Prescher, S. Hessler. The sensory innervation of the hip joint-an anatomical study. Surg Radiol Anat.vol.9,pp.37-375,1997.

[13] P. Louie, E. Lucchinetti, L. Zhang, A. Affolter, M.Schaub, M.Gandhi. The Mechanism of Intralipid-Mediated Cardioprotection Complex IV Inhibition by the Active Metabolite, Palmitoylcarnitine, Generates Reactive Oxygen Species and Activates Reperfusion Injury Salvage KinasesSocSciMed.vol.202,pp.38-42, 2018.

[14]X. Capdevila, C. Coimbra, O. Choquet. Approaches to the lumbar plexus: success, risks, and outcome.Regional anesthesia and pain medicine.vol.30,pp.50-62, 2005.

[15]MJ. Barrington, R. Kluger. Ultrasound guidance reduces the risk of local anesthetic systemic toxicity following peripheral nerve blockade. Regional anesthesia and pain medicine.vol.38,pp.289-9, 2013.

[16]H. Elsharkawy, K. El-Boghdadly, M. Barrington. Quadratus lumborum block: anatomical concepts, mechanisms, and techniques. Anesthesiology.vol.30,pp.322-35, 2019.

[17] S. D. Adhikary, K. El- Boghdadly, Z. Nasralah, N. Sarwani, A. M. Nixon K. J. Chin. $\mathrm{A}$ radiologic and anatomic assessment of injectate spread following transmuscular quadratus lumborum block in cadavers. Anaesthesia.vol.72,pp. 73-79, 2017.

[18] M. Wikner. Unexpected motor weakness following quadratus lumborum block for gynaecological laparoscopy.Anaesthesia.vol. 72,pp.230-32, 2017.

[19]P. Kukreja, L. Macbeth, A. Sturdivant Anterior quadratus lumborum block analgesia for total hip arthroplasty : a randomized, controlled study. Reg Anesth Pain Med 2019.

[20] M. A. L. Desmet Balocco, V.Van Belleghem. Fascia iliaca compartment blocks: different techniques and review of the literature. Best Pract. Res. Clin. Anaesthesiol 2019.

[21] D.Bugada, V. Bellini, L. F. Lorini, E. R. Mariano. Update on Selective Regional Analgesia for Hip Surgery Patients. Anesthesiol. Clin.vol.36,pp. 403-5, 2018.

[22] H.Nassar, A.Hasanin, M.Sewilam, H.Ahmed, M.Abo-Elsoud,O.Taalab, H.A. Zoheir, 
Transmuscular Quadratus Lunborum Block Versus suprainguinal Anesthesia.vol.4,pp.6790,2012.

[23] J. Hashmi, B. Cusack, L. Hughes,ESRA9-0226 Comparing analgesic efficacy of quadratus lumborum block versus fascia iliaca block in patients undergoing total hip replacements .Regional Anesthesia \& Pain Medicine.vol.44,pp. 06-07, 2019.

[24] Y. Aoyama, S. Sakura, S.Abe, S. Tadenuma, Y. Saito. Continuous quadratus lumborum block and femoral nerve block for total hip arthroplasty: a randomized study. J Anesth.vol.34,pp.43-20,2020.

[25] R.Blackwell, M.Kushelev, J0.Norton, R.Pettit, w.k. Vasileff,. Acompartive Analysis of the Quadratus lumborum and fascia Iliaca Biocks in Hip Arthroplascopy,sports medicine ,and rehabilitation.vol. 3,pp.7_3, 2012.

[26] T. Parras, R. Blanco. Randomised trial comparing the transversus abdominis plane block posterior approach or quadratus lumborum block type I with femoral block for postoperative analgesia in femoral neck fracture, both ultrasound-guided. Rev Esp Anestesiol Reanim.vol.63,pp.4-8,2016.

[27]JG. Xing, FW. Abdallah, R. Brull. Preoperative femoral nerve block for hip arthroscopy: A randomized, triplemasked controlled trial. Am J Sports Med.vol.43,pp. 2680-87, 2015.

[28] MQ.Potter ， GS. Sun, JA. Fraser, JT Beckmann, JD. Swenson, TG. Maak. Psychological distress in hip arthroscopy patients affects postoperative pain control. Arthroscopy.vol.30,pp.95-20,2014.

[29] H.Ueshima, O.Hiroshi. Incidence of lowerextremity muscle weakness after quadratus lumborum block. J Clin Anesth.vol. 44,pp.0-4, 2018. 